On the vibrational behaviour of cyanide adsorbed at $\mathrm{Pt}(111)$ and $\mathrm{Pt}(100)$ surfaces in alkaline solutions

\title{
F. Huerta
}

Dept. Ingeniería Textil y Papelera. Universidad Politécnica de Valencia. Paseo Viaducto, I E-03801 Alcoy (Spain)

\section{F. Montilla}

Instituto de Biologia Molecular y Celular. Universidad Miguel Hernández de Elche. Avda. de la Universidad s/n E-03202 Elche (Spain)

E. Morallón, J. L. Vázquez

Dept. Química Fisica. Universidad de Alicante. Apartado 99. E-03080 Alicante (Spain)

* Corresponding author.

e-mail: jl.vazquez@ua.es

Fax: (+34) 965903537

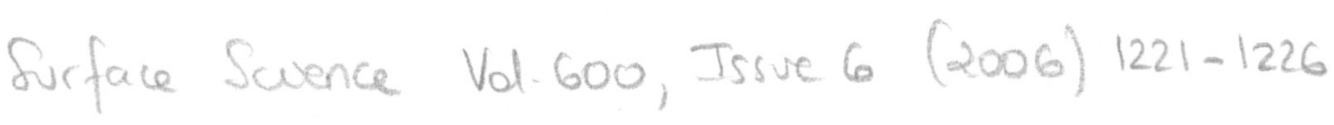




\begin{abstract}
This communication deals with the vibrational behaviour of cyanide adlayers formed on $\mathrm{Pt}(111)$ and $\mathrm{Pt}(100)$ surfaces in the electrochemical environment. In situ FTIR spectroscopy can be employed to follow the potential dependence of the $\mathrm{C}-\mathrm{N}$ stretching frequency in acidic electrolytes with quite a low uncertainty. Owing to the extreme stability of the cyanide adlayer in alkaline solutions, parallel experiments performed in $\mathrm{NaOH}$ medium are usually perturbed by the significant overlapping of the reference and the sample FTIR spectra. Deconvolution of the spectra assuming a Lorentz oscillator allowed to confirm that for Pt(111)-CN in perchloric acid medium, two potential regions with different band center frequency tuning coexist. Conversely, in the alkaline electrolyte a single tuning rate for the band position was found for both surfaces studied. The lack of reorientation of the C-N molecular axis toghether with the occurrence of a certain screening effect of negatively charged hydroxyl anions on the electric field at the interface could be at the origin of the different behaviour displayed in both electrolytic media.
\end{abstract}

Keyword s: infrared spectroscopy; single crystal electrodes; cyanide; adsorption; Stark effect; 


\section{Introduction}

The in situ vibrational spectroscopies have been employed to study many model molecules adsorbed at the electrochemical interface. In particular, much attention has been paid to the intriguing metal-cyanide interaction. Cyan ide ions are able to adsorb on almost all the metal electrodes forming a very stable, strongly-bonded adlayer which has been characterized under ultra-high vacuum conditions by great number of ex situ techniques [1-4]. One of the main interesting topics related to the CN-metal interaction is the experimental observation that the C-N vibrational frequency depends linearly on the electrode potential. This has been explained in two different ways: either as a change in the molecule's chemical bonding or as the effect of the interfacial electric field on the molecule, usually referred to as the electrochemical Stark effect $[2,5-8]$.

It is frequently observed that the in situ vibrational experiments on the metal$\mathrm{CN} \mid$ solution interface are complicated by other interesting effects, such as the occurrence of (electro)chemical reactions [5,6,9-11], the reorientation of the molecular axis [7-9,12] or the displacement of the molecule between adsorption sites [4,13,14]. Since some cyanide reactions, such as the formation of soluble $\mathrm{Pt}-(\mathrm{CN})_{\mathrm{x}}$ complexes or the oxidation of the adlayer, seem often favoured by polycrystalline substrates, the problem has been usually overcome taking advantage of single crystal electrodes. In addition, single crystals provide unique two-dimensional order that facilitates the interpretation of the vibrational information. A singular exception to this rule was observed when the CN-Pt(100) system was examined by in situ FTIR spectroscopy in acidic medium $[9,15]$. It was reported that there exists only a narrow potential region for which the cyanide adlayer is stable, being oxidised to NO or decomposed to $\mathrm{CO}$ above and below the stability region, respectively. It is also known that adsorbed cyanide can occupy either on-top or bridged sites when the adlayer is formed, for example, at $\mathrm{Pd}[16,17]$ and $\mathrm{Ag}[18]$ surfaces but the on-top configuration seems always preferred at Pt electrodes. In spite of this, the potential-induced reorientation of the C-N molecular axis with respect to the surface plane has been reported to occur when the cyanide adlayer is formed on roughened Pt electrodes [7]. Similar interpretation has been also employed to explain the existence of two different infrared absorption bands for the Pt 111 )CN system [9]. As a final point the movement of adsorbed $\mathrm{CN}^{\top}$ to new adsorption sites in acidic medium is one of the most interesting observations in relation to this adsorbate, matn if we take into account the supposed strength of the bond between Pt(111) and cyanide. The 
phenomenon reported in the literature involves a potential dependent structural transition from a $(2 \sqrt{3} \times 2 \sqrt{3}) \mathrm{R} 30^{\circ}-\mathrm{CN}$ to a $(\sqrt{7} \times \sqrt{7}) \mathrm{R} 19^{\circ}-\mathrm{CN}$ arr angement in perchloric acid solutions [13].

Both Raman and infrared spectroscopies have been widely app lied to the investigation of the potential dependence of the cyanide $\mathrm{C}-\mathrm{N}$ stretching frequency. Raman experiments performed at polycrystalline substrates can provide valuable information on the cyanide frequency change against the applied potential because the subtraction of a reference spectrum is unnecessary. In spite of this advantage, the intensity of the recorded signal does not contain any quantitative information and, besides, this technique cannot be applied to single crystal electrodes. On the contrary, infrared spectroscopy has been extensively used as a surface probe for single crystals in the electrochemical environment even though the processing of the single beam spectrum is always required. In order to obtain absolute absorption bands, one should process two single beam spectra, one of which contains the vibrational information and the other should be collected in the absence of the adsorbate. Otherwise, the absorption band will always appear as a bipolar feature and no quantitative information could be derived. In this context, the present work is devoted to the spectroscopic characterisation of cyanide adlayers formed on Pt(111) and Pt(100) surfaces. The vibrational behaviour will be examined in both perchloric acid and sodium hydroxide solutions.

\section{Experimental}

Cyanide adlayers were obtained by immersion of a clean Pt(111) or Pt(100) electrode into a $0.1 \mathrm{M} \mathrm{NaCN}$ solution (Merck p.a.) under open circuit conditions for 2-3 min. The electrode was then rinsed with ultrapure water and transferred to the spectroelectrochemical cell. In these conditions, the coverage obtained for adsorbed cyanide is near to saturation. The solutions employed for the in situ FTIR spectroscopy experiments were either $0.1 \mathrm{M} \mathrm{HClO}_{4}$ (Merck Suprapur) or $0.1 \mathrm{M} \mathrm{NaOH}$ (Merck, p.a.). All potentials were measured against a reversible hydrogen electrode (RHE) immersed in the test solutions and are presented in this scaie. The spectrometer employed for the in situ FTIR experiments was a Nicolet Magna 850 equipped with a nitrogen cooled MCT detector. The spectroelectrochemical cell was provided with a prismatic $\mathrm{CaF}_{2}$ window bevelled at $60^{\circ}$. Spectra were collected at $8 \mathrm{~cm}^{-1}$ resolution and at room temperature. They are presented in the usual form $\Delta R / R$. 


\section{Results and discussion.}

Fig. 1 shows two sets of in situ FTIR spectra collected for a Pt(111) electrode covered with an adsorbed cyanide layer in $0.1 \mathrm{M} \mathrm{HClO}_{4}$ and $0.1 \mathrm{M} \mathrm{NaOH}$ solutions, both free of bu $\mathrm{k}$ cyanide. The processed spectra are displayed after the normalized subtraction of 100 sample interferograms (which were acquired at the sample potentials ind icated in the figure) and 100 reference interferograms (collected at $0.1 \mathrm{~V}$ for each series). It is clearly observed that the spectra obtained in the whole potential range yield always a bipolar band for the C-N stretching mode at around $2100 \mathrm{~cm}^{-1}$. The bipolar character of the feature reflects that not only the sample spectra but also the reference one contain some vibrational contribution of the cyanide stretching mode. In order to obtain undisturbed information about the potential dependence of both band centre position and intensity of the vibration, it would be strongly desirable that the absorption band under investigation was not distorted by the reference spectrum. To register the absolute bands for adsorbed cyanide, a reference spectrum was obtained in acidic media at $1.5 \mathrm{~V}$. Since the cyanide adlayer is completely oxidised at such positive potentials, the series of in situ FTIR spectra obtained at sample potentials between 0.1 and $1.1 \mathrm{~V}$ in perchloric acid medium can be obtained in the form of monopolar bands (at around $2100 \mathrm{~cm}^{-1}$ ) corresponding to the C-N stretching of ad sorbed cyanide [9].

However, the scenario is quite different in alkaline medium because the cyanide adlayer cannot be fully oxidised within the stability window of the system. Therefore, it is not possible to obtain a set of monopolar absorption bands as it has been previously performed for the acidic electrolyte. To overcome the difficulty, bipolar features in Fig. $1 \mathrm{~b}$ have been deconvoluted assuming a Lorentz-type absorption. The maximum experimental separation between both peaks is obtained at $0.9 \mathrm{~V}$. Hence, the deconvolution of both bands can be performed at this potential with the lowest uncertainty. For the process to be completed, the constant positive-going b and was calculated from that spectrum corresponding to adsorbed cyanide at the reference potential $(0.1 \mathrm{~V})$. In this way, its frequency $\left(207^{7} \mathrm{~cm}^{-1}\right)$ is assumed to keep constant during the collection of all the subsequent spectra of the series. It is worth noting that the assumption is strictly correct because a unique reference spectrum was collected for the complete set of specta. The peak position of the positive-going band was calculated after deconvolution of the spectrum obtained at the higher potential $(0.9 \mathrm{~V})$. This strategy was followed in order to ach ieve the maximum separation between positive-going and negative-going bands. The lorentzian curve obtained was subtracted from the total 
spectrum at each potential and the results are displayed in Fig. 2. The quality of the deconvolution method was assessed comparing the calculated spectra with the experimental results obtained with the reference spectrum collected at $1.5 \mathrm{~V}$ in acidic medium. Fig. $3 \mathrm{a}$ shows the dependence of both the peak frequency and the integrated band intensity on the applied potential for the calculated negative-going bands in Fig. 2a. It is observed that the band centre frequency shifts with a positive tuning rate of $105 \mathrm{~cm}^{-1} \mathrm{~V}^{-1}$ at potentials between $0.1 \mathrm{~V}$ and $0.5 \mathrm{~V}$, but only with $25 \mathrm{~cm}^{-1} \mathrm{~V}^{-1}$ at potentials positive to $0.5 \mathrm{~V}$. At the same time, the evolution of the integrated band intensity with the potential fits well with the experimental data reported previously for the Pt(111)-CN system, for which a tuning rate of $100 \mathrm{~cm}^{-1} \mathrm{~V}^{-1}$ was obtained at potentials lower than $0.5 \mathrm{~V}$ and a $30 \mathrm{~cm}^{-1} \mathrm{~V}^{-1}$ tuning rate was measured above that potential [9]. Therefore, the accuracy of the mathematical treatment is confirmed by the excellent correspondence obtained for the experimental and deconvoluted spectra in acidic medium. At this point, it was assumed that the mathematical treatment could be applied with similar success to the alkaline electrolyte. In this way, the spectra of Fig. $1 \mathrm{~b}$ were processed in an analogous way to that employed for the acidic medium and the series of calculated spectra are displayed in Fig. 2b. For this series it is observed that, in parallel to the results obtained in acidic conditions, the negative-going band shifts to higher frequencies with the applied potential. The evolution of the band centre position with the applied potential is depicted in Fig. $3 b$, from which a positive tuning rate of $40 \mathrm{~cm}^{-1} \mathrm{~V}^{-1}$ can be derived. The integrated band intensities follow similar trend, showing a continuous increase with the potential from $0.2 \mathrm{~V}$ to $0.9 \mathrm{~V}$ in the alkaline electrolyte.

From the results presented is clearly derived that the $\mathrm{C}-\mathrm{N}$ stretching vibration behaves differently in acidic and alkaline electrolytes. On the one $h$ and, in perchloric acid medium the existence of two potential regions for the potential dependence of the band centre frequency, specifically those above and below $0.5 \mathrm{~V}$, has been clearly observed. Conversely, in the alkaline electrolyte there exists a single tuning rate for the band position whose value, $40 \mathrm{~cm}^{-1}$ $\mathrm{V}^{-1}$, is found between those observed in acidic medium above and below $0.5 \mathrm{~V}$. In terms of the vibrational Stark effect, the lower potential dependence of the band centre frequency below $0.5 \mathrm{~V}$ seems to indicate that the local electric field which actually $\mathrm{h}$ as an effect on the cyanide adsorbed molecules is less dependent on the potential drop across the double layer in the $\mathrm{OH}^{-}$ containing electrolyte than in the acidic medium. In other words, the existence of a certain screening effect of negatively charged hycroxyl anions could be at the origin of the different behav iour displayed in both electrolytic media at potentials less positive than $0.5 \mathrm{~V}$. 
As mentioned above, it has been also proposed in the literature that cyanide could adsorb through the $\mathrm{N}$ atom in acid medium in the lower potential region, whereas the molecular axis could be inverted at potentials higher than $0.5 \mathrm{~V}$. However, the behaviour of the C-N stretching frequency for cyanide adsorbed in alkaline medium suggests that no rearrangement of the adlayer takes place upon polarization of the electrode either above or below $0.5 \mathrm{~V}$, since a uniform tuning rate is observed in the whole potential range. Attending to the low value of the tuning frequency, the most probable orientation of the cyanide adlayer in alkaline medium is that for which the $\mathrm{C}$ end is directed tow ards the Pt substrate and the $\mathrm{N}$ end oriented to the solution, similarly to the conformation displayed by the adsorbed layer in acidic medium at potentials above $0.5 \mathrm{~V}$. However, the N-bound configuration cannot be fully discarded on this surface since the variation of the integrated band intensity follows a similar trend to that found for the adsorbed layer in acidic medium at potentials below $0.5 \mathrm{~V}$. For the $\mathrm{N}$-bound cyanide the probability of the vibrational transition rises as the potential is made more positive. Further studies must be performed in order to precise the actual effect of the $\mathrm{OH}^{-}$in the behaviour of adsorbed cyanide on this surface. On this basis, the lack of reorientation of the adsorbed layer in alkaline medium could account for the different behaviour observed in $\mathrm{H}^{+}$and $\mathrm{OH}^{-}$containing electrolytes.

A similar study was performed with the Pt(100)-CN system. In situ FTIR spectra were collected for a Pt(100) electrode covered by a cyanide adlayer in alkaline solution $(0.1 \mathrm{M}$ $\mathrm{NaOH}$ ) free of bulk cyanide. Fig. 4a shows the spectra collected at different sample potentials (reference spectrum acquired at $0.1 \mathrm{~V}$ ). Bipolar bands around $2100 \mathrm{~cm}^{-1}$ can be observed as in the case of the $\mathrm{Pt}(111)-\mathrm{CN}$ system. $\mathrm{CN}$ ad layers are stable in alkaline medium in the potential range between $0.1-1.1 \mathrm{~V}$. A negative band appears around $2165 \mathrm{~cm}^{-1}$ with a low in tensity. The intensity of his band grows with the potential and can be clearly observed at 1.1V. This band corresponds to $\mathrm{C}-\mathrm{N}$ stretching vibrations of cyanate ions formed by the oxidation of the adsorbed cyanide layer. This result contrasts with the behaviour of the $\mathrm{Pt}(100)$-CN adlayer in acidic solutions, since the stability window of the aclay er in perchloric acid is restricted to the $0.4 \mathrm{~V}-0.6 \mathrm{~V}$ range. Above those potentiais ad sorbed CN is oxidised to $N O$ and at potentials lower than $0.4 \mathrm{~V}$ the adlayer reacts to fom adsorbed Co [15].

In orde: to obtain a monopolat band in the potential range O.1-1.1V the same mathematical treatment was applied to the experinentel results. The evolution of the band 
center and integrated intensity with the applied potential are shown in Fig. 4b. A single tuning rate for the band center frequency can be calculated for the whole potential range of $43 \mathrm{~cm}^{-1}$ $\mathrm{V}^{-1}$, which is a value very similar to that obtained with the Pt(111)-CN system in alkaline solutions. However the evolution of the band intensity is opposite showing a continuous decrease between 0.2 and $1.1 \mathrm{~V}$, as can be observed in Fig. $4 \mathrm{~b}$. On the basis of our previous discussion the trend of the evolution of the C-N stretching frequency suggests that no rearrangement of the adlayer takes place upon polarization of the electrode in the whole potential range. The value of the tuning frequency is very similar in both surfaces studied (near $40 \mathrm{~cm}^{-1} \mathrm{~V}^{-1}$ ) indicating that the surface structure of the adlayer has a low influence in the electroch emical Stark effect of the CN adsorbate. Both, the low tuning frequency value and the decreasing intensitiy of the absorption band with the applied potential for $\mathrm{CN}$ adlayers formed on $\mathrm{Pt}(100)$ are similar to the trends observed for $\mathrm{Pt}(111)-\mathrm{CN}$ in acidic media at potentials above $0.5 \mathrm{~V}$. Therefore, the most probable orientation of the cyanide adlayer in alkaline medium on the $\mathrm{Pt}(100)$ electrode is that for which the $\mathrm{C}$ end is directed towards the substrate and the $\mathrm{N}$ end oriented to the solution.

\section{Conclusion.}

Cyanide adlayers formed on $\mathrm{Pt}(111)$ and $\mathrm{Pt}(100)$ surfaces have been examined by in situ FTIR spectroscopy in alkaline solutions. Assuming a Lorentz-type oscillator, the deconvolution of the $\mathrm{C}-\mathrm{N}$ stretching absorption bipolar bands has been performed. The accuracy of the mathematical treatment has been checked against the $\mathrm{Pt}(111)-\mathrm{CN} \mid \mathrm{HClO}_{4}$ system for which, is easy to experimentally obtain absolute absorption bands. The good accordance between experimental and calculated values allowed to extend the mathematical model to both $\mathrm{Pt}(111)-\mathrm{CN} \mid \mathrm{NaOH}$ and $\mathrm{Pt}(100)-\mathrm{CN} \mid \mathrm{NaOH}$ systems in order to obtain the potential dependence of both band center frequency and integrated band intensity. Thus, it has been observed that in the alkaline electrolyte a single tuning rate for the band position was found for both surfaces studied. The lack of reorientation of the $\mathrm{C}-\mathrm{A}$ molecular axis toghethet with the accurrence of a certain screening effect of negativ ely charged hydroxyl anions on the electric field at the interface could be at the origin of the different behaviour displayed in both electrolytic media. 


\section{Acknowledgements}

Dr. F. Montilla thanks the Spanish Ministerio de Educación y Cultura for the award of his Juan de la Cierva contract. Financial support from the Ministerio de Educación y Cultura (MAT2004- 01479) and Generalitat Valenciana (GV5/136) is gratefully acknowledged.

\section{References}

[1] J.L. Stickney, S.D. Rosaco, G.N. Salaita, A.T. Hubbard, Lan gmuir 1 (1985) 66.

[2] P.L. Hagen, X. Guo, I. Chorkendorff, A. Winkler, H. Siddiqui, J.T. Yates, Jr, Surf. Sci 203 (1988) 1.

[3] N.A. Booth, R. Davis, D.P. Woodruff, D. Chrysostomou, T. McCabe, D.R. Lloyd, O. Schaff, V. Fernandez, S. Bau, K.-M. Schindler, R. Lindsay, J.T. Hoeft, R. Terborg, P. Baumgärtel, A.M. Bradshaw, Surf. Sci. 416(1998) 448.

[4] M. Lennart, M. Arenz, C. Stuhlmann, K. Wandelt, Surf. Sci. 461 (2000) 98.

[5] C.S. Kim, C. Korzeniewski, J. Phys. Chem. 97 (1993) 9784.

[6] W. Daum, K.A. Friedrich, C. Klünker, D. Knabben, U. Stimming, H. Ibach, Appl. Phys. A 59 (1994) 553.

[7] B. Ren, X-Q. Li, D-Y. Wu, J-L. Yao, Y. Xie, Z-Q. Tian, Chem. Phys. Lett. 322 (2000) 561.

[8] B. Ren, D-Y. Wu, B-W. Mao, Z-Q. Tian, J. Phys. Chem. B 107 (2003) 2752.

[9] F.J. Huerta, E. Morallón, J.L. Vázquez, A. Aldaz, Surf. Sci. 396 (1998) 400.

[10] K. Ashley, D.L. Feldheim, D.B. Parry, M.G. Samant, M.R. Philpott, J. Electroanal. Chem. 373 (1994) 201.

[11] K.A. Friedrich, W. Daum, C. Künker, D. Knabben, U. Stimming, H. Surf. Sci. 335 (1995) 315 .

[12] A. Tadjeddine, A. Peremans, A. Le Rille, W.Q. Zheng, M. Tadjeddine, J.P. Flament J. Chem. Soc. Faraday Trans. $92(1996) 3823$.

[13] Y-G. Kim, S-L. Yau, K. Itaya, I. Am. Chem. Soc. 118 (1996) 393. 
[14] F. Ample, A. Clotet, J.M. Ricart, Surf. Sci. 558 (2004) 111.

[15] F. Huerta, E. Morallón, J,L. Vázquez, J. Electro anal. Chem. 480 (2000) 101.

[16] K. Ashley, F. Weinert, M.G. Samant, H. Seki, M.R. Philpott, J. Phys. Chem. 95 (1991) 7409 .

[17] K. Ashley, M. Lazaga, M.G. Samant, H. Seki, M.R. Philpott, Surf. Sci. 219 (1989) L590.

[18] M. Fleischmann, I. R. Hill, M. E. Pemble, J. Electroanal. Chem. 136 (1982) 361. 


\section{Figure Captions}

Fig. 1. Sets of in situ FTIR spectra obtained for a Pt(111) dosed with a cyanide adlayer in either (a) $0.1 \mathrm{M} \mathrm{HClO}_{4}$ or (b) $0.1 \mathrm{M} \mathrm{NaOH}$ electrolytes. Reference potential $0.1 \mathrm{~V}$. Sample potentials indicated for each spectrum in the figure. p-polarized radiation. 100 interferograms collected at each potential

Fig. 2. Calculated absorption bands for the C-N stretching of cyanide adsorbed on $\mathrm{Pt}(111)$ as a function of the applied potential in $0.1 \mathrm{M} \mathrm{HClO}_{4}$ (a) and $0.1 \mathrm{M} \mathrm{NaOH}$ (b) electrolytes. Deconvolution was performed assuming a Lorentz oscillator.

Fig. 3. Potential depend ence for the calculated peak frequencies and band intensities of the C$\mathrm{N}$ stretching in $0.1 \mathrm{M} \mathrm{HClO}_{4}$ (a) and $0.1 \mathrm{M} \mathrm{NaOH}$ (b). Data derived from Fig. 2.

Fig. 4. (a) Experimental FTIR absorption bands collected for the C-N stretching of cyanide adsorbed on $\mathrm{Pt}(100)$ as a function of the applied potential in $0.1 \mathrm{M} \mathrm{NaOH}$. Reference acquired at $0.1 \mathrm{~V}$. p-polarized radiation. 100 in terferograms at each potential; (b). Potential dependence for the calculated band center frequency and band intensity of the C-N stretching in $0.1 \mathrm{M}$ $\mathrm{NaOH}$. Deconvolution performed assuming a Lorentz o scillator. Data derived from (a). 


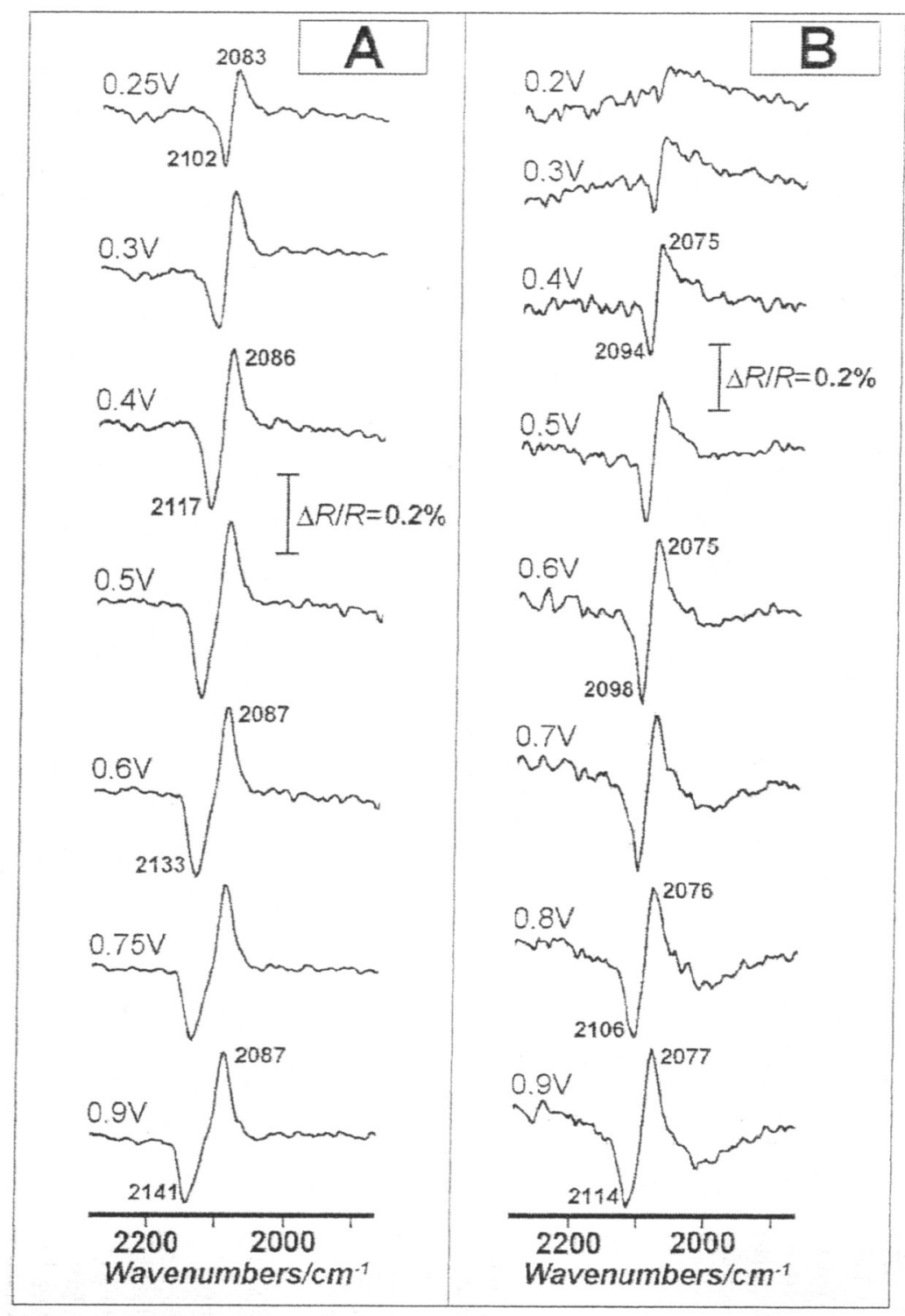

Fig1a

1b 


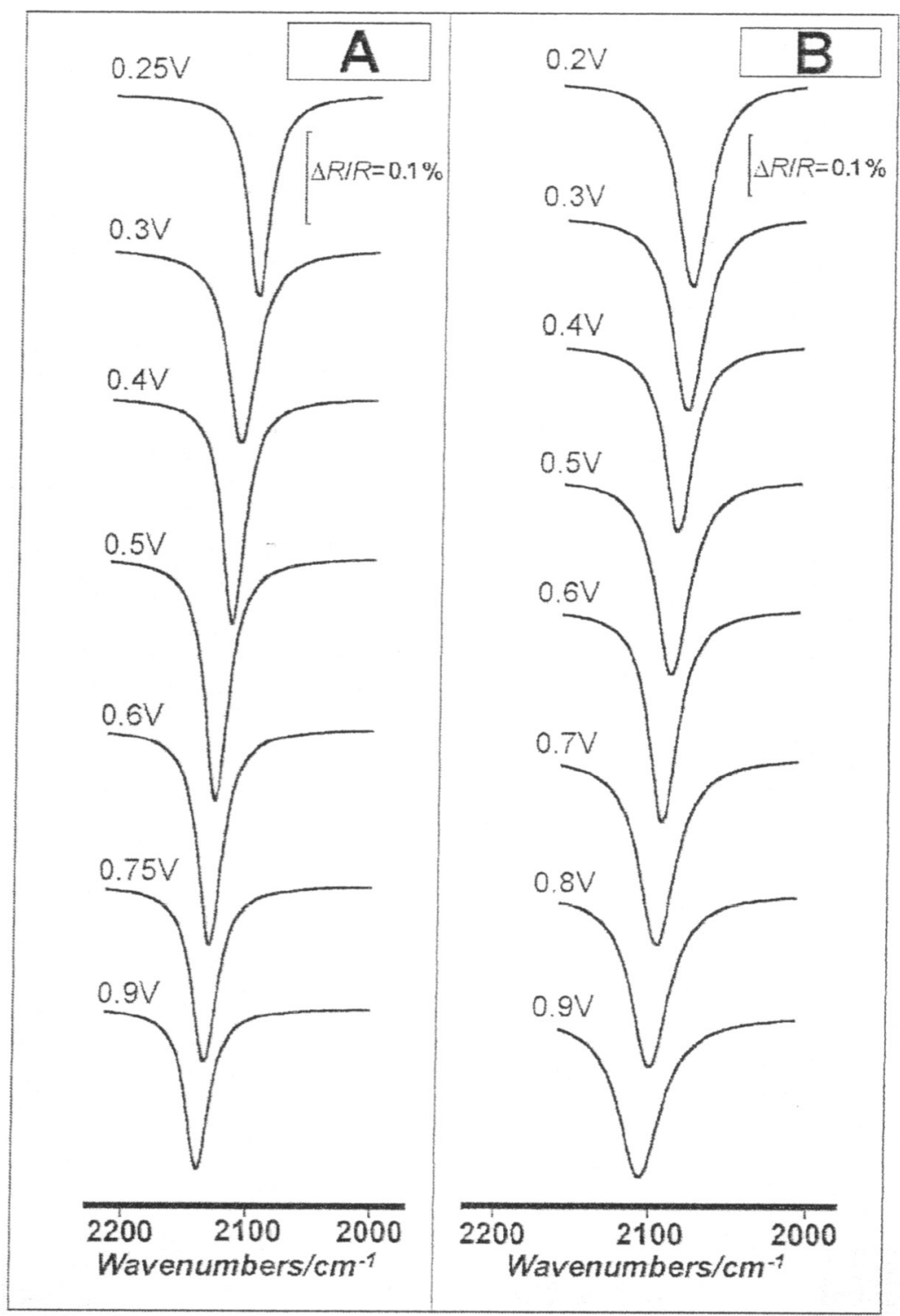

Fig2a

2b 


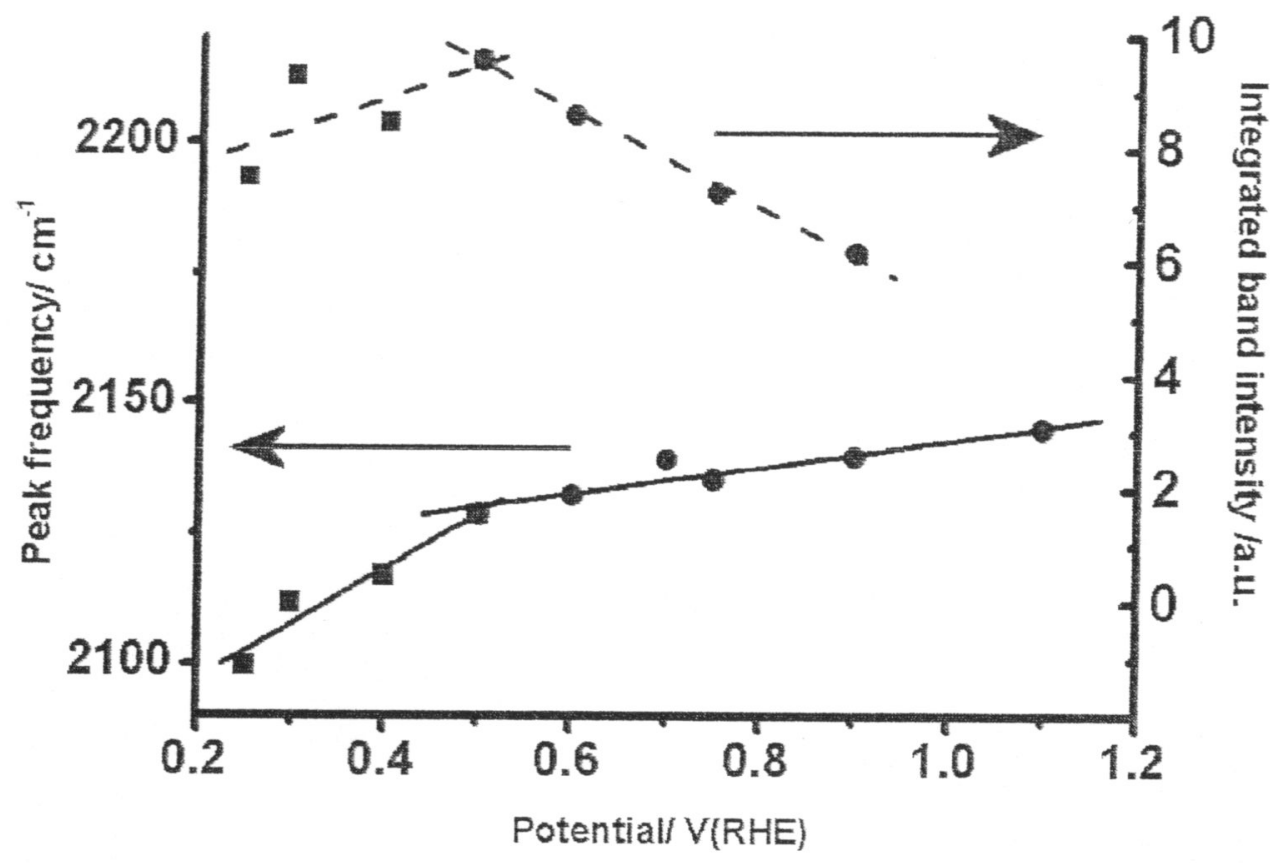

Fig 3.a

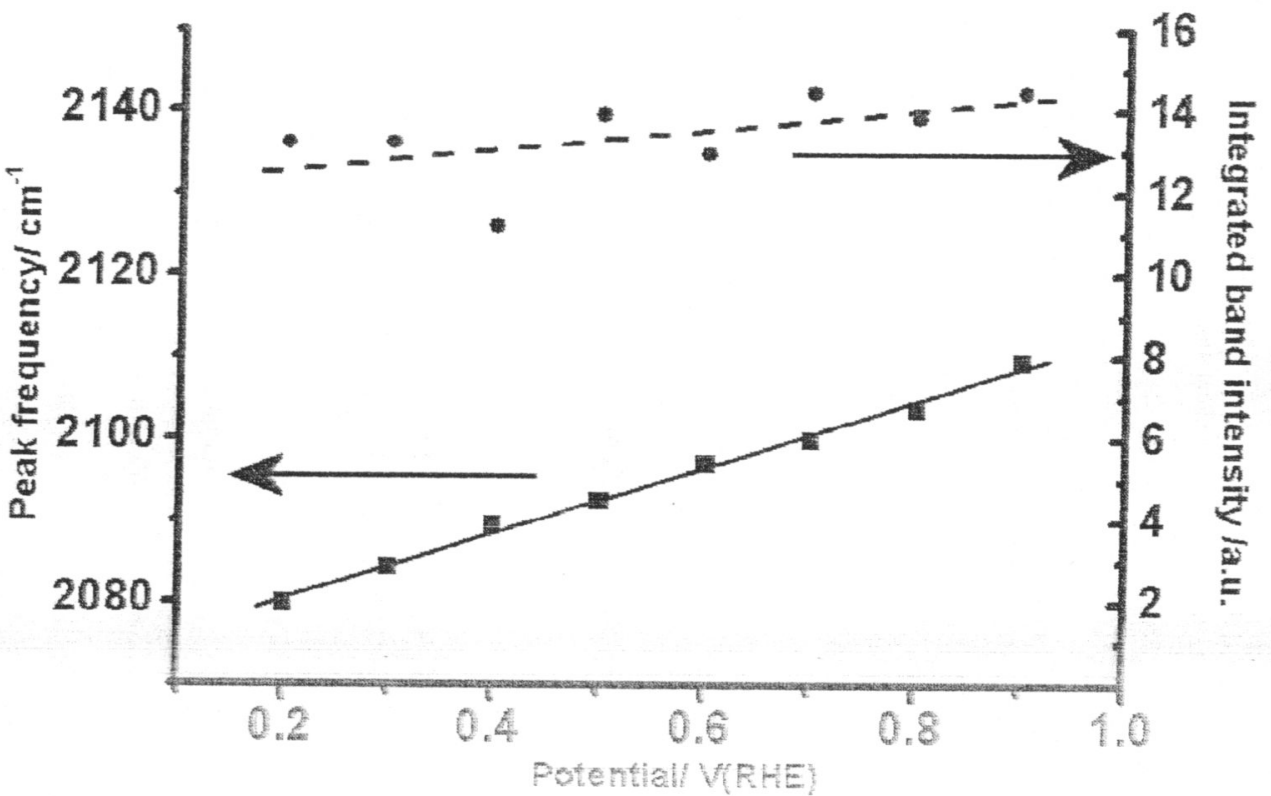

Fig $3 b$ 


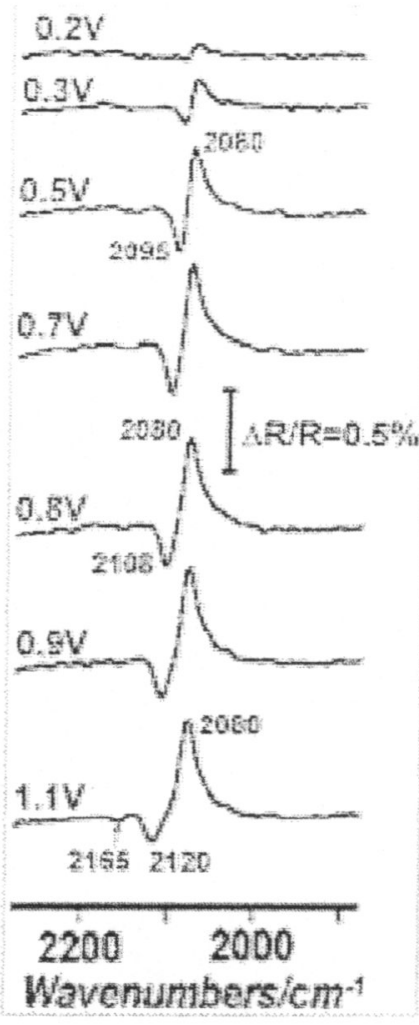

Fig 4.a

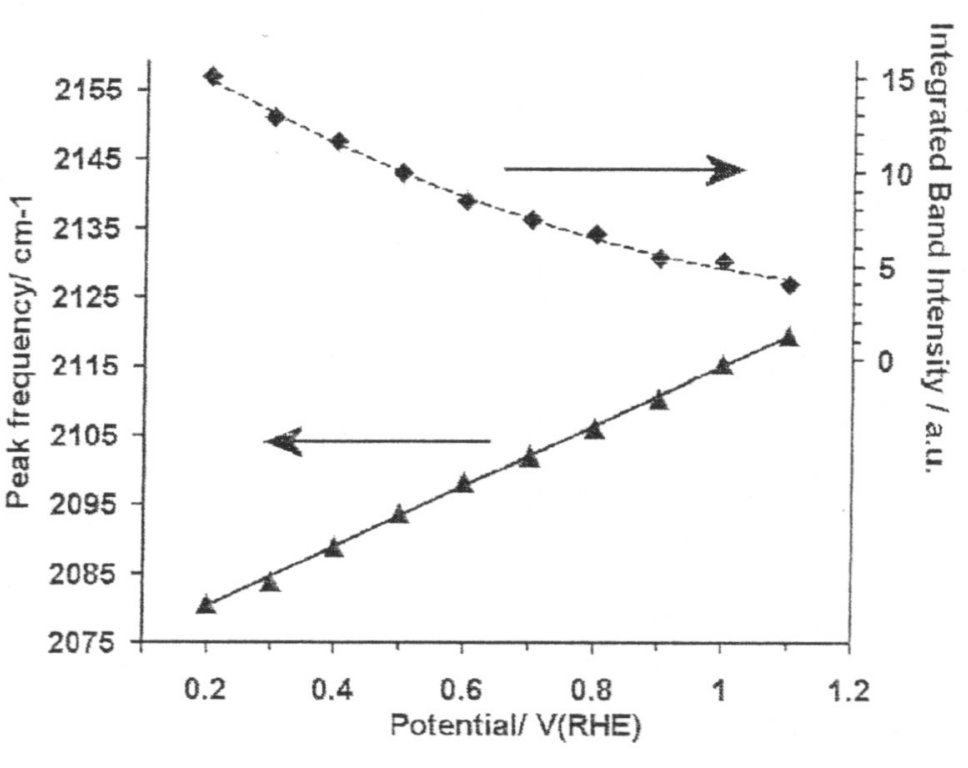

4.6 